Atomic lifetime measurements of highly charged ions

E. Träbert

May 27, 2008

Handbook for Highly Charged Ion Spectroscopic Research 
This document was prepared as an account of work sponsored by an agency of the United States government. Neither the United States government nor Lawrence Livermore National Security, LLC, nor any of their employees makes any warranty, expressed or implied, or assumes any legal liability or responsibility for the accuracy, completeness, or usefulness of any information, apparatus, product, or process disclosed, or represents that its use would not infringe privately owned rights. Reference herein to any specific commercial product, process, or service by trade name, trademark, manufacturer, or otherwise does not necessarily constitute or imply its endorsement, recommendation, or favoring by the United States government or Lawrence Livermore National Security, LLC. The views and opinions of authors expressed herein do not necessarily state or reflect those of the United States government or Lawrence Livermore National Security, LLC, and shall not be used for advertising or product endorsement purposes. 


\title{
Atomic lifetime measurements of highly charged ions
}

\author{
Elmar Träbert \\ Astronomisches Institut, Ruhr-Universität Bochum, D-44780 Bochum, Germany, \\ and \\ High Temperature and Astrophysics Division, Lawrence Livermore National \\ Laboratory, Livermore, CA 94550-9234, USA \\ E-mail: traebert@astro.rub.de
}

\begin{abstract}
.
Transition probabilities, transition rates, Einstein A values all denote the same, a measure of the likelihood that a transition between two atomic energy levels takes place. If there are several decay paths from a given level, the $\mathrm{A}$ values indicate the relative importance of the individual paths. The inverse of the sum of all radiative decay rates is called the (radiative) level lifetime. Atomic level lifetimes can be more easily measured than the more fundamental, but experimentally often less accessible transition rates.

The transition rates tell about allowed and dis-allowed ('forbidden') decay processes which are characterized by different dependences on the nuclear charge $Z$ or the ion core charge $\zeta$. Varying $Z$ (the element) along an isoelectronic sequence permits systematic studies of various contributions to atomic structure and dynamics. With highly charged ions, level lifetimes have been studied from the femtosecond range to many seconds, employing a wide variety of measurement techniques that encompasses ion beams and stored ions, passive and active interrogation.

The major experimental techniques are being discussed along examples of what can be learned about atomic structure and dynamics. In any environment, the level lifetime has to be compared to the collision time to see the competition of radiative and collisional processes. The competition can affect the lifetime measurement, but it can also reveal important information on the environment, for example, on temperature and density.
\end{abstract}

to be Submitted to: Y. Zou, R. Hutton, F. Currell, I. Martinson, S. Hagmann (Eds.):

"Techniques for Highly Charged Ion Spectroscopy

PACS numbers: $32.70 . \mathrm{Cs}, 32.30 . \mathrm{Jc}, 39.30 .+\mathrm{w}$ 


\section{Introduction}

Atomic line spectra reveal atomic structure and the fact that energy is quantized. Only transitions between the 'fixed' excitation levels of an atom are possible. However, not all combinations of levels occur in actual spectra, and not all spectral lines are of similar intensity. These observations point to selection rules (invoking parity, angular momentum, spin, etc.), and to the concept of transition probability, or the "A factor" $\mathrm{A}_{k i}$ for a transition from level $\mathrm{k}$ to level $\mathrm{i}$. The mean life $\tau$ that appears in the exponential decay law of an excited level is the reciprocal of the sum of all transition probabilities from a given level:

$$
\tau_{k}=1 / \Sigma\left(A_{k i}\right)
$$

Atomic lifetime measurements on electric dipole (E1) transitions yield information on atomic wave functions that supplements the insight gained from atomic energy levels alone. The E1 transition rate depends on the transition energy and an extra power of $r$, because the electric dipole operator er explicitly depends on $r$, the vector from the nucleus to the position of the electron. Transitions between fine structure levels of a given term ("forbidden transitions", magnetic dipole (M1) and electric quadrupole (E2) transitions) are supposedly insensitive to this, as they connect levels with similar radial wave functions. However, complex wave functions as well as relativistic effects in highly charged ions modify this simple picture.

Interpreting $\tau$ as the time constant of a damped oscillator, there are two ways to measure this parameter. Firstly, by the (Lorentzian) line width, or, secondly, by measuring the line intensity as a function of time and fitting an exponential curve to the data. (Lorentzian line profile and exponential decay curve are Fourier transforms of each other.) Classical spectroscopy is hardly sufficient to observe the natural line width of atomic levels. Narrowband laser spectroscopy, however, has achieved this goal. Also, with (nanosecond-) pulsed laser excitation, it is nowadays quite feasible to selectively excite and then measure the typical nanosecond lifetimes of most low-lying levels of neutral atoms and of some levels of singly charged ions. This option pertains to levels that decay by electric dipole (E1) radiation.

E1 transition rates of transitions which involve a change of principal quantum number $n(\Delta n \neq 0)$ scale as $Z^{4}$. For multiply charged ions the level lifetimes therefore are much shorter than for neutral ions. They are out of the reach of lasers both because of the laser photon energy being insufficient to reach the excited levels and excite them selectively, and because the decay time usually is too short for classical electronic timing measurements. An alternative is provided by fast ion beams that experience excitation when being passed through a thin foil [1]. The ions in the beam lose a fraction of their energy, but apart from that, the ion beam leaves the foil largely unharmed and continues its trajectory. Distance of the ions from the rear side of the foil translates into time after the end of excitation. Therefore one can record the spatial decrease of the light intensity emitted by the ion beam as a function of distance from the foil and convert that to a time measurement in the picosecond to many-nanosecond range. 
Atoms with levels that are particularly short-lived may have a natural line width that is greater than the Doppler and instrumental line widths that are typical for beam-foil spectroscopy. If autoionization is a direct competitor to the radiative decay one observes, then the intensity of the radiative branch and thus the signal rate suffers, of course. Fortunately, there are cases in which the lower level of a transition autoionizes and thus broadens the final level, whereas the radiative signal benefits from an unbranched radiative decay. Given the typical autoionization rates of the order of $10^{14} \mathrm{~s}^{-1}$, the typical lifetimes studied in this way are in the range of a few femtoseconds (for examples see [2,3]).

Electron beam ion traps intuitively suggest that lifetimes to be studied there would be long (else there would be no need for extended storage). We will discuss how such measurements of long lifetimes in the microsecond to many-millisecond range can be done with electron beam ion traps and electronic timing. We will also explain how short lifetimes (again in the femtosecond range) can be addressed by a line width measurement, and how all these techniques relate to other types of lifetime measurement, for example, by beam-foil spectroscopy. Reviews of measurements of long atomic lifetimes in various types of ion traps have been presented elsewhere $[4,5,6]$.

With so much work already done, and theory providing atomic structure properties (including level lifetimes) cheaply (on the basis of atomic structure algorithms that need only seconds of run time on modern personal computers), is it really worth doing such experiments? Ever so often it has, indeed, been suggested to leave all further atomic lifetime work to theory. For the multitude (tens of thousands) of high-lying levels and the vast number (hundreds of thousands) of transitions between them that are required in viable collisional-radiative models which are used to provide line intensity references for specific plasma observations, theory can't be replaced by experiment, because of the sheer quantity of the necessary data and the practical inaccessability of most decays. However, besides this vast amount of levels and transitions that are not needed to be known individually with high accuracy, there usually are some key transitions that need to be known well and that can be measured and should be measured as a benchmark for the theoretical model. Sometimes it is found that experiment has underestimated systematic errors, sometimes theoretical results are clearly inappropriate. In the interplay of experiment and theory, both can and do evolve. Mutual challenges are very helpful in this context.

Occasionally it has been suggested that theory is now so good that surely no further effort (money) should be wasted on atomic lifetime experiments. I have heard such comments from an eminent theoretician when I began to measure a particularly longlived class of levels. Decades earlier the same person had been named as living proof for the superiority of theory (and she has done excellent work). In my case, I was happy to report that I found - by way of experiment - some calculations by this eminent expert to be very good, indeed, and others far from satisfactory. The latter happened to be for transitions that this very person had stated as needing no experimental work, because the calculations were so good already. Much of what used to be atomic 
structure theory has migrated to theoretical chemistry, where thousands of molecules are handled in amazing detail by enormous computations. However, the techniques are different. A theoretical chemistry colleague heard of our intercombination transition lifetime measurements and offered to solve the problem by using their superb algorithms. The results took much longer than anticipated and never came close to our experimental data. An additional note of caution will be reflected in the 'Examples' section: Until very recently, the fine structure intervals in multiply charged multi-electron ions were not reliably calculated by anyone. Seeing this, a customary resort was to replace the calculated energy splitting by the experimentally much better known one, before calculating the transition rate. Only in the last few years have $a b$ initio calculations been successful in deriving such splittings and then transition probabilities for electric-dipole forbidden transitions without having to resort to 'semi-empirical' corrections. Such complex computations may take weeks on a present-day personal computer, including checks for convergence and reliability. And a last note: Calculations that come after the experimental fact should not be considered as having proven any predictive power. Key atomic lifetime experiments will continue to be of high value.

\section{Experimental techniques}

The concepts of A values (Einstein coefficients), oscillator strengths $f$ and line strengths $S$ are about a century old, predating the actual capability to measure atomic level lifetimes. The first techniques aiming for this goal employed the absorption of light to derive oscillator strengths, and exploited the insight that absorption $\left(f_{i k}\right)$ and emission $\left(f_{k i}\right)$ oscillator strengths between lower level $\mathrm{i}$ and upper level $\mathrm{k}$ are equal (but the statistical weights $g=2 J+1$ have to be taken into account). Atomic absorption spectroscopy is still a viable analytic technique, and occasionally absorption may still be used to determine very small oscillator strengths. In astrophysics, absorption spectra dominate - but they are rarely, if ever, used to determine lifetimes. Nowadays emission techniques dominate, especially so since photoelectric detection has enabled linear measurements of signal strengths over wide dynamic ranges. Progress in vacuum technology has made it possible to produce and, if necessary, store ever more highly charged ions. Progress in technical tools has made it possible to produce and ever more selectively excite such ions. A variety of techniques to measure atomic lifetimes has been developed over the years, but they all belong to just a few categories that exploit two aspects of radiative decay.

A) The natural line width is related to the upper level lifetime.

B) If excitation suddenly stops, an excited level will decay exponentially, with the level lifetime $\tau$ appearing as the time constant of the exponential $d N / d t=A N_{0} e^{-\left(t-t_{0}\right) / \tau}$, where $N_{0}$ is the upper level population at time $t=t_{0}$ and $d N / d t$ is the decay rate. $\mathrm{A}$ and $\mathrm{B}$ are mathematically equivalent, implying that a Lorentzian line profile (with a certain width) is the Fourier transform of an exponential function. In practice, line widths can only be measured if they are sufficiently large (very short-lived levels, say, in 
the femtosecond lifetime range). Exponential decays have been measured from the range of a few femtoseconds to the range of minutes, but this wide range cannot be measured by one and the same technique. The techniques actually used are mainly based on electronic timing (from the nanosecond range upward) or on spatial measurements on fast ion beams, translating lateral displacement to time via the speed of the ions.

Historically, some of the techniques have produced excellent results with ions in low charge states or with neutral atoms. However, referencing all the good work done there would overburden the present chapter, and I'll restrict my presentation to multiply and highly charged ions. I'll thus leave out, for example, the excitation of atomic beams by a beam of electrons or laser light, pulsed excitation of gases by energetic electron beams, or a detailed description of various tricks employed in the laser excitation of beams of singly charged ions. In multiply charged ions, the first excitation step (from the ground state or a low-lying metastable level) is usually too large to be bridged by present-day lasers, but synchrotron radiation may step into the gap at some stage, and therefore the principles will be discussed below. The very coarse ordering principle of the techniques presented below is by the atomic level lifetime range covered, using either ion beams or ion trapping, or both. Of the line width measurements there are very few, and I will discuss them in the context of the overarching beam-foil and electron beam ion trap work.

2.1. Fast ion beams: Beam-foil, beam-laser, beam-gas-laser, beam-foil-laser, line width measurement of autoionization

Ion accelerators started out as devices for nuclear physics, sending energetic particles at others that often were exposed to the ion beam in the form of thin foils or as a coating on a thin foil of, for example, carbon. The target foil needs to be thin so that the energy loss of the fast ions inside the foil is not so large as to wash out the energy dependence of a nuclear excitation resonance or whatever is being measured. Reaction products are to be measured for their energy, which is another reason to keep target foils thin. This, however, implies that the original ion beam is minimally affected and largely travels on unharmed, that is, at about the same energy (speed) and in the same direction as before. In several laboratories people noticed that there was light emission from the ion beam, but only two researchers $[7,8,9,10,11,12]$ realized the atomic physics options that were opened by this beam-foil technique (sketched in figure 1): Excitation of whatever single elemental species, effected at an adjustable energy, and the time distribution of atomic decays being drawn out in space. (In the 1920s, Wilhelm Wien had had some key ideas along these lines, but his experimental plans were far ahead of the necessary vacuum and other technologies.)

In terms of atomic lifetime measurements, there would be a discussion of what time interval describes the excitation process, whether it is the transit time through the foil or any time scale associated with interactions near the rear surface of the foil. The only cleanly defined time interval seemed to be the transit time spent inside the foil. At a 


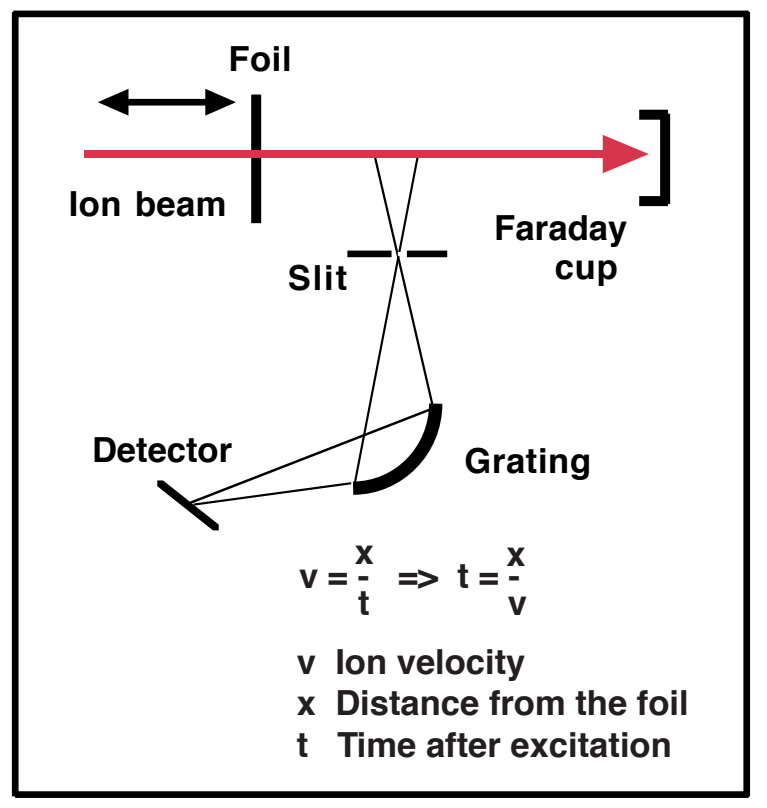

Figure 1. Schematics of beam-foil spectroscopy.

mass density of $10 \mu \mathrm{g} / \mathrm{cm}^{2}$, a carbon foil is about $30 \mathrm{~nm}$ thick, and a typical ion beam of energy $0.5 \mathrm{MeV} / \mathrm{amu}$ (atomic mass unit), that is $500 \mathrm{keV}$ protons or $14 \mathrm{MeV}$ Si ions, needs about 3 fs to traverse this foil. At the time, no ions with valence electron lifetimes of this order of magnitude were of interest or produceable. However, the interaction of the ions with the foil material was a matter of discussion, whether the excitation process of swift ions was dominated by collisions with the atomic cores of the foil material atoms or with the bound or quasi-free electrons. This question was studied by observing $\mathrm{x}$ rays from inside the foil and outside the foil, that is, emitted by ions with (short-lived) innershell vacancies. The vacancy lifetimes were estimated to be compatible to the transit time, and it was found that with thicker foils the x-ray yield increased. This effect was eventually exploited to model ionization and electron capture - and to derive $\mathrm{K}$ vacancy level lifetimes in the femtosecond range [13]. This was a demonstration of principle, but had no chance to yield a precise measurement. A major obstacle there lies in the fact that it was (and is) difficult to measure accurately the thickness (and density) of such foils (which are about $1 / 800^{\text {th }}$ the thickness of ordinary writing paper).

Beam-foil spectroscopy has emerged as a generic name for many techniques that make use of fast ion beams for atomic physics (and 'fast-beam spectroscopy' might be a less restrictive title). All of these use the interaction of the fast ion beam with a medium (solid or gas) to establish a charge state distribution and provide excitation. However, a laser might be used instead of the material target foil or in addition to it. I will start from the basic arrangement and then explain some of the variants.

In the basic scheme, an ion beam of whatever energy (traveling in a high vacuum environment) passes through a thin foil. Depending on the beam energy, the ions may capture or lose electrons, and a new charge state distribution emerges that depends 
mostly on the ion energy; its width may also depend on the foil material (for simplicity and handling, the standard material is carbon, but beryllium, aluminium, gold and whatever else have also been employed). The ions experience a statistically distributed small energy loss and also some angular scattering. In the early years of beam-foil spectroscopy, when the ion beam energies were rather moderate, much effort was spent on characterizing nuclear vs. electronic energy loss (from collisions with the screened nuclear Coulomb field of the target foil ions or with the quasi-free electrons), but at higher energies, the first part becomes negligible, as does large-angle scattering, the process by which ions deviate from the beam trajectory and get lost. Because of the mass ratio, the collisions with the light electrons are strongly peaked forward (smallangle scattering). The energy loss in matter (which is important also for various nuclear physics experiments) has been extensively studied, parameterized and tabulated [14]. However, as stated before, the thickness of a foil cannot routinely be determined with good accuracy, and therefore an energy-loss correction of the ion speed by a few percent may well itself be uncertain within a sizable fraction of the correction (say 10 to $20 \%$ ). Moreover, under ion irradiation, crystallographic reordering can take place and change foil properties like the areal density.

It is best to measure the ion energy after the interaction with the target. For light ion beams with energies up to a few hundred $\mathrm{keV}$, a magnet or an electrical sector field can be employed to deflect the ion beam and to control the beam energy via feedback to the accelerator. For high energy ion beams, this effort is impractical. In some accelerator laboratories that operate pulsed ion beams, time-of-flight techniques have been implemented. To make such measurements precise, a long flight path is required, which usually precludes implementation after the ions have passed through an exciter foil at the experiment. Since the beam velocity is a crucial part of the lifetime measurement using beam-foil excitation (see below), the uncertainty of the ion velocity makes for a serious limitation of the lifetime measurement precision. It is no surprise then that the decisive factor in the most accurate beam-foil lifetime measurement reported so far [15], on neutral helium atoms, has employed an in-beam technique to establish the time scale, in this case by concurrently observing a well-calculated quantum beat pattern in the decay of another level of the same atomic species, which serves as a built-in atomic clock. Such a quantum beat frequency, however, increases with the fine structure splitting, which increases with the fourth power of the nuclear charge $Z$. In highly charged ions the spatial frequency of the quantum beats is therefore too high to be practically employed for reference there.

Following the above example of $0.5 \mathrm{MeV} / \mathrm{amu}$, a typical ion beam speed is about $1 \mathrm{~cm} / \mathrm{ns}$ (light travels $30 \mathrm{~cm}$ in a nanosecond), or $10 \mu \mathrm{m}$ per picosecond. Owing to the constant velocity of the ions after leaving the exciter foil, distance from the foil is strictly proportional to time after excitation. A detector can simply travel along the excited ion beam and register the light intensity (suitably filtered or observed through a spectrometer, see figure 1). The spatial curve relates to a development in time. A spatial displacement of the field of view by $1 \mu \mathrm{m}$ (easily achieved mechanically) corresponds to 
a time interval of $100 \mathrm{fs}$ - and no high-speed clock is required to measure atomic lifetimes of only a few picoseconds [16].

The minimum mechanical displacement interval, however, does not define the minimum lifetime that can be measured. This has several reasons. The ion beam usually has a diameter of several millimeters, and the exciter foil is not necessaily flat over the full beam cross section. A detector viewing the ion beam sideways normally captures a divergent cone of light, not a parallel pencil of light, and thus very fine spatial structures of the decay curve are smeared out in the observation. In order to collect enough light so that the signal can exceed the detector noise, the field of view at the location of the ion beam should not be too narrow. This intrinsic integration over a section of the ion beam favors the observation of longer lifetimes over the very short ones, that is, of decays that die out before the ions have even passed through the full field of view. (For a similar reason, a wide field of view would integrate over many quantum beat oscillations, making the contrast suffer.) However, the width of the field of view influences the relative intensity collected of a decay component, but has a limited influence on the time resolution. For that, the detail of the function that represents the field of view matters, that is, the wings of the usually trapezoidal 'window function' are essential for probing any spatial / temporal detail [17, 18, 19, 20]. Taking this feature into account, lifetimes have routinely been measured down to a few picoseconds.

However, time resolution is a problem that limits the range of objects to study. The transition rate of electric dipole transitions between shells scales with $Z^{4}$. In highly charged ions most of the levels that have such decay channels are too short-lived for practical measurements. These very transitions are basically the same as in hydrogenlike ions, and thus they can be calculated with confidence - there is no expectation that any foreseeable measurement could reach the accuracy necessary to test the quality of the calculations. The interest in employing a window function in most cases is somewhat different from time resolution: The very fact of a structured detection zone distorts the purely exponential decay curve (usually a superposition of several exponentials, dur to cascade repopulation). Any evaluation without a window function has to cut off the data channels of highest signal or suffer systematic error. With a complete curve analysis (taking the part that is affected by the window function into account), this waste is avoided, and the actual foil position (time zero) can be recovered as a bonus. This knowledge is helpful when evaluating the relative intensities of the various decay components, and is providing physically meaningful constraints in the modeling of complex decays.

A much more important problem than time resolution is posed by complexity. The very advantage of beam-foil spectroscopy, the fact that practically any atomic level can be excited in collisions under high-density conditions, has the attached downside of all of these levels deexciting and thus repopulating lower levels the decay of which may be of immediate interest in a given measurement. Hence all beam-foil lifetime measurements deal with multi-exponential decays. A number of techniques have been derived which aim at selective excitation of the level of interest (seeking a single-exponential decay 
curve, which is much easier to evaluate), as will be discussed below. In straightforward BFS, there will be complex decay curves, but some of the experimental situations yield access to decay curves of manageable complexity, and these cases are of great practical importance.

Least-squares fitting of exponential curves to decay data is non-linear (i.e., there is no mathematical inversion process that leads to a unique solution); a systematic variation of the parameters is undertaken to minimize the (quadratic) deviation of a (synthetic) fit curve from the data. In parameter space, minima of a hypersurface are being sought, for various models (one exponential plus background, two exponentials plus background, etc.), with success (small $\chi^{2}$ value) being measured in relation to the statistical scatter of the data. It can be shown how certain combinations of amplitudes and lifetimes of only two decay components result in data curves that cannot be analyzed reliably. Even the combination of a single exponential with a flat detector background poses serious problems, if the decay curve does not comprise a sufficiently long section of background after the decay has died out. Decay curves well represented by three exponentials can be analyzed, if the time constants of the exponentials differ from each other by at least a factor of three. Analyses with more than three exponentials usually remain ambiguous, unless one or more lifetimes and/or amplitudes can be constrained on the basis of other measurements or theoretical insight. It helps, if the shorter-lived components have the relatively larger amplitudes - this situation corresponds to about equal initial level populations.

The cleanest technique of analyzing decay curves for the lifetime of a specific level requires not only the measurement of the decay of that level, but also of all cascades into that level, so that they can be subtracted out from the decay curve of interest. This ANDC technique (Arbitrarily Normalized Direct Cascades) [21, 22, 23] does not need to measure the actual cascade transitions, but can work with other decay branches of the same feeding levels, because all decay curves of a given level have the same pattern (plus individual background levels of the individual detectors). In cases with two or three dominant cascades (such as the nsnp ${ }^{1} \mathrm{P}_{1}^{\mathrm{o}}$ levels in $\mathrm{Be}-$ or $\mathrm{Mg}$-like ions), the ANDC technique has been highly successful. In the face of cascade level lifetimes very close to that of the primary decay, the technique has recovered the correct primary lifetime with good accuracy. In contrast, a naive multi-exponential fit of these curves returns a result that is systematically too long by 30 to $50 \%$. The high fraction of BFS results with exactly this error situation illustrates the need for ANDC analysis whenever possible, as well as a historic lack of attention to atomic structure detail in a number of laboratories which has become apparent in systematic error studies [24, 25].

Unfortunately, ANDC is not always feasible, because several or all of the major cascades may occur in a different spectral range (for example, x-ray vs. EUV) for which no spectrometer is available locally. Also, the cascade level lifetimes may be so short, that only the cascade tail (a number of cascade steps away from the wanted direct cascade) can be measured with sufficient time resolution, reducing the veracity of the cascade input to ANDC. In such cases, cascade modeling based on semi-empirical 
assumptions about level populations can provide an approximation that at least is better than a fit of too few exponentials to the original data. Cascade modeling would benefit from a small set of population parameters that describe the $n$ - and $l$-dependence of the level population after ion-foil interaction. However, the search for generally valid simple population laws has not met with success.

Since the inter-shell transitions (see above) are so hydrogen-like, fast, and better calculated than measured, lifetime measurements using beam-foil spectroscopy have largely dealt with level lifetimes that for one reason or another are much longer. Examples are intercombination transitions in He-like ions (at rather low $Z$ up to about $Z=16$ ), the magnetic dipole decay of the $1 s 2 .{ }^{3} \mathrm{~S}_{1}$ level (from $Z=16$ to $Z=54$ ), and the 2E1, M1, and M2 decays of other $n=2$ levels in one- and two-electron ions, up to $Z=92$ [26]. Next there are the $\Delta n=0$ resonance transitions in Li-like (up to $Z=92$ [27]), Na-like, and Cu-like ions, resonance and intercombination transitions in Be- [28], Ne-, Mg-, Al-, Si- [29], Zn-, Ga-, and Ge-like ions, and so on. A number of these sequences have been systematized by Curtis who also has found ways to combine resonance and intercombination transition rates in a joint representation [30]. Such work is very helpful in detecting inconsistencies and systematic errors, and ultimately establishes the basis for consistent pictures of our knowledge of atomic structure and dynamics.

The measurement of these relatively long-lived level lifetimes is actually helped by cascades. The dominant cascade pattern in most cases may be seen as one of a single electron outside a core, that is, a hydrogenic model. The branching ratios of most decays favour a change of the orbital angular momentum quantum number $l$ by -1 , and the energy scaling favours a maximum change of principal quantum number $n$. This results in an evolution of the level population towards the yrast line of levels of maximum $l$ for a given $n$. Once there, the further decays need many steps of $\Delta n=1$ to reach the low-lying levels. In highly charged ions, the low-lying excited levels are very short lived, while high-lying yrast levels can be very long-lived. Thus a pattern emerges: the low-lying excited levels quickly repopulate the possibly long-lived levels one is interested in and practically empty the associated reservoir; this population boost enhances the signal that one observes from the decay of level of interest. However, there always is a tail of many slow yrast contributions, and it is worth including this tail in an analysis [31], although the individual cascade amplitudes are very small. The superposition of those many slow exponential contributions with their underlying steady progression of lifetimes can be described by a power law, and a time dependence such as $t^{-1.5}$ has been repeatedly observed [32].

The cascade problem in lifetime measurements could be avoided, if selective excitation of only the level of interest was possible. In atoms, of course, single or multiple laser excitation is a standard technique. There also are schemes for combining fast atom beams and lasers, producing the fast atoms from a beam of singly charged ions that capture electrons from a dilute gas target; such an isotopically pure beam of same-velocity atoms has certain advantages over experiments in which a laser is pointed 
at a gas cell. For rare gas atoms, the electron capture offers the excitation of metastable levels which are rather lying too high for most lasers. Visible laser light would then excite the atoms from there to resonance levels, and the subsequent decay to the ground state can be monitored almost free of background contributions in the vacuum ultraviolet. Such schemes have also been applied to multiply charged ions, for example, starting from the metastable $2 s$ level of one-electron ions and seeking to induce by resonance with laser light the transition to one of the $2 p$ levels, in the quest for accurate Lamb shift determinations. Short-wavelength light at high power levels is available at synchrotron light sources. In fact, in one experiment synchrotron light was employed to ionize and excite Ar, and a lifetime of a level in $\mathrm{Ar}^{+}$was obtained with high accuracy [33]. Possibly this approach will some day reach multiply ionized species.

Only one experiment, however, appears to have combined foil-excitation with subsequent laser excitation of an ion in order to measure atomic level lifetimes [34]. This experiment on two levels of singly charged $\mathrm{N}^{+}$demonstrates important points. The beam-foil interaction results in a shift and broadening of the velocity distribution in the beam, which makes it difficult to exploit high-resolution laser techniques. The level is being excited by the ion-foil interaction even without the help of the laser. The experiment is therefore less clean than one might imagine under "selective excitation": decay curves obtained without the laser are subtracted from decay curves obtained with the laser on resonance (each of which have statistical scatter). The result was a single exponential decay, as was hoped for, but it was difficult to achieve, facing such problems as the sensitivity of the frequency match for resonant excitation of fast ions (Doppler effect) whose velocity depends on (changing) properties of the exciter foil. In multiply charged ions, most level splittings exceed by far the photon energies of practical lasers, and then the selective excitation by laser resonance is just out of reach. In highly charged ions, however, forbidden transitions in the ground configuration, or even the hyperfine splitting of the lowest levels of very highly charged few-electron ions, can be large enough so that laser techniques are of interest again - and will be discussed in the section on heavy-ion storage rings.

There is one very different lifetime measurement technique that involves fast ion beams, although the primary role is that of the ion-foil interaction which amply populates also multiply excited states. When observing the light emission of an ion beam at the rear surface of the exciter foil, especially in the EUV and x-ray ranges, there is almost a continuum of radiation from very short-lived core-excited ions. (The not so short-lived ones among them, for example, the Li-like ions in the $1 s 2 s 2 p{ }^{4} \mathrm{P}_{5 / 2}^{\circ}$ state, can be studied by standard foil-displacement measurement techniques.) Moving away from the foil, the spectrum rapidly gets cleaner, with fewer and fewer lines surviving. However, there exist curious cases of ions which decay to an autoionizing state which hence is extremely short-lived and thus broadened, and the lower-level broadening can be seen in the line width of the transition leading to the respective states. Fast-beam observations with a fast spectrometer (large solid angle of acceptance) usually suffer notable Doppler broadening, which for some spectrometer designs can be countered by 
refocusing [35, 36, 37, 38, 39] which, unfortunately, is likely to distort the line profile somewhat. Observations of the line broadening due to the shortening of a level lifetime by autoionization $[2,3]$ had results in the ball park $\left(10^{-14} \mathrm{~s}\right)$ that theory predicted, however, the remaining disagreement was never fully resolved as to which part might be blamed on experimental problems and which on the shortcomings of theory.

\subsection{Slow ion beam: Recoil ions}

Beam-foil spectroscopy encounters a physical limit when striving for the study of very long-lived levels, in the sheer size of the decay path associated with that. When Marrus and Schmieder $[40,41,42]$ attempted to measure the about 200-ns lifetime of the $1 s 2 s$ ${ }^{3} \mathrm{~S}_{1}$ level in the He-like ion $\mathrm{Ar}^{16+}$, their $8 \mathrm{MeV} / \mathrm{amu}$ ion beam from SuperHILAC traveled about $8 \mathrm{~m}$ per atomic lifetime. It is difficult to control the geometry of an ion beam over such distances, with energy and angle straggling in the exciter foil adding to the problem. Moreover, a given decay happens only once per ion, and thus the signal per unit of ion beam path is very low for long-lived levels. Under these circumstances, it is quite understandable that Marrus and Schmieder did not recognize a major systematic error, the presence of core-excited ions with a spectator electron [43], that caused their measurement to yield a lifetime $15 \%$ short of expectation. (Work by the same group years later found a result that agreed with calculations - theory also needed some sorting out.) Intrigued by the problem of such long level lifetimes, my own group made two attempts to employ slower beams. One attempt called for beamfoil production and excitation of the right charge state (requiring an ion beam energy of about 2 to $3 \mathrm{MeV} / \mathrm{amu}$ ) and then substantially decelerating the ion beam (in this case, $\mathrm{Cl}^{15+}$, with a calculated lifetime of about $400 \mathrm{~ns}$ ) in a post-accelerator switched to deceleration mode. Such an energy variation by more than an order of magnitude had been demonstrated at the Heidelberg MPI-K institute, but our atomic physics experiment missed the time window before the TSR storage ring was being built (which later on permitted to measure lifetimes many orders of magnitude longer). Our other approach [44, 45, 46] employed a beam of highly charged ions at GSI Darmstadt. A primary beam of highly charged uranium ions at an energy of $1.4 \mathrm{MeV} / \mathrm{amu}$ was passed through an Ar gas target where it produced a wide range of mostly low-energy (few-eV) recoil ions. With a weak electric field, ions were extracted sideways and then accelerated to about $1 \mathrm{keV}$ per charge (figure 2 ). The only long-lived level capable of emitting x-rays was the $1 . s 2 .{ }^{3} \mathrm{~S}_{1}$ level in the He-like ion $\mathrm{Ar}^{16+}$, and its $\mathrm{x}$-ray decay was monitored by a 10-cm long position sensitive detector along the recoil ion beam path - a detector idea proposed decades earlier by $\mathrm{J}$ R Mowat. The recoil ion beam was then charge-separated in a magnetic sector field, and the x-rays that were detected in (delayed) coincidence with the correct charge state ions were counted, the events being stored as a function of position (corresponding to flight time) in the x-ray detector. The pilot experiment

reached an accuracy of about $5 \%$; its results were compatible with later fast-beam work at Berkeley. Incidentally, photon-ion coincidences have been applied very recently with 


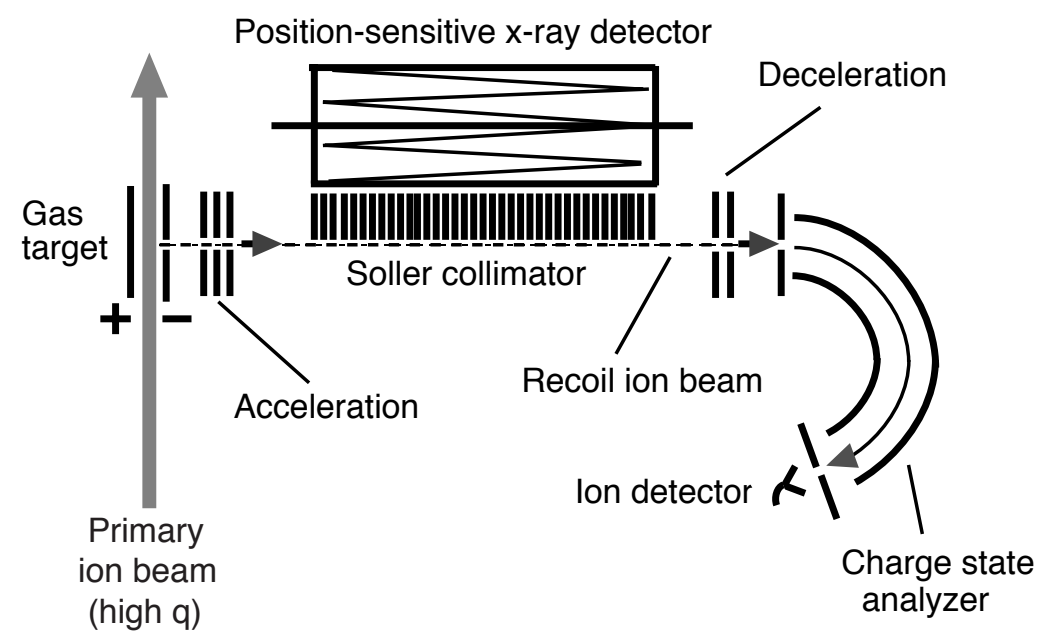

Figure 2. Slow ion beam ion-photon coincidence schematics (adapted from [1]).

fast ion beams, too: The lifetime of the hyperfine-quenched $1 s 2 p{ }^{3} \mathrm{P}_{0}$ level in the Helike ion of $\mathrm{Au}(\mathrm{Z}=79)$ was measured at GSI Darmstadt, separating the ion charge states after the photon observation, and cleaning the x-ray spectrum by filtering out only those $\mathrm{x}$-ray events that coincided with the right ion charge state ions [47].

Nowadays such an ion-photon coincidence measurement scheme for long-lived atomic lifetimes (in the microsecond range) would profitably be set up using the relatively slow ion beam available from an electron cyclotron resonance ion source (ECRIS) or an electron beam ion source (EBIS). Such an arrangement would yield experimental access to atomic lifetimes in between the beam-foil (straight fast ion beam) experiments discussed above, which can reach up to about $100 \mathrm{~ns}$, and the ion trap experiments (discussed below) which work best from many microseconds to many seconds, although measurements down to $700 \mathrm{~ns}$ [48] have been reported. Depending on the spectral range of the emitted radiation, the position-sensitive x-ray detector in the above scheme would have to be replaced by some detector for the EUV or UV range. Position-sensitive EUV detectors of the size of the x-ray detector may be rather expensive; the equipment cost could be lowered at the expense of measurement time by placing a regular detector (or a small, fast spectrometer) a traveling mount that can be displaced alongside the recoil ion trajectory. Such a scheme may remind the reader of the basic lay-out of beam-foil spectroscopy - but at velocities (specific energies) that are about two (four) orders of magnitude lower. The decisive point is the decoupling of the production of highly charged ions from the ion beam energy, whereas in beam-foil spectroscopy the two are strongly linked.

\subsection{Stored ion beam: Heavy-ion storage ring}

At level lifetimes of a few hundred microseconds, fast ions travel about a kilometer during one such lifetime. A thread of yarn or a wire of great length are easily stored 
if rolled on a spool. The same can be done with fast ion beams. Bending the ion beam trajectory around to form an approximate circle, the same elements of the beam guidance system are used over and over again. From one turn to the next, the trajectory is slightly shifted, so that by this stacking procedure a beam current can be accumulated that (at TSR Heidelberg) is higher than the original one by up to a factor of thirty. Then the injection into the storage ring vessel is stopped, and the beam can be left cruising, or be further accelerated, or be cooled by (partial) superposition of a cold (low energy spread) electron beam. Depending on the ion charge state and energy, and, most importantly, on the extremely good vacuum $\left(10^{-14} \mathrm{bar}\right)$, the beam can last for seconds, minutes, or hours. The circumference of the TSR heavy-ion storage ring TSR at Heidelberg is $55 \mathrm{~m}$, and the ions typically need a few microseconds per turn. Other such storage rings (ASTRID at Aarhus, CRYRING at Stockholm) are rather similar in size, ESR at GSI Darmstadt and the new Lanzhou facility in China are twice as large. Various schemes of lifetime measurements have been employed at heavy-ion storage rings. Conceptually the simplest is to use the ions as they are provided by the injecting accelerator (figure 3). That machine uses a gas or foil stripper to produce ions in high charge states, and there are many levels of interest in such ions that have lifetimes way beyond the time the ion beam needs to travel to the storage ring (some $5 \mu \mathrm{s}$ ) and to settle down in a stable beam configuration after injection and stacking (less than $1 \mathrm{~ms}$ ). All one has to do is to synchronize the data recording cycle to the ion injection and to store the signal of a stationary detector as a function of time. The ions will pass by the detector every few $\mu \mathrm{s}$, and the signal rate will go down over time with the number of ions surviving in excited states. There are no moving parts and no geometry changes (as there are in BFS) during the measurement. Detectors for photons can be mounted outside the storage ring vessel, observing the ion beam through a window, or inside (for EUV radiation). In this way a great number of intercombination transitions in light ions and E1-forbidden transitions in the ground configurations of Fe-group ions $[49,50,51,52,53]$ have been studied at Heidelberg, with lifetimes from $0.5 \mathrm{~ms}$ [54] to some $50 \mathrm{~s}[55]$.

Most of these lifetimes are shorter than the process of electron cooling would require (several seconds at least), which is why electron cooling has not been used in most measurements. However, the electron beam of the electron cooler (or a second such device acting as an electron target) can be tuned away from the ion velocity to an energy that brings the electrons in dielectronic resonance (DR) with the ions. Then inner excitation can take place, and the ion can bind an electron and form a multiply excited system that either reverts to its previous state (giving up the former beam electron) or stabilize radiatively. Then the ion has changed charge state and will be caught by a specific detector after the next bending magnet. The cross sections for the DR process are so small that the DR signal acts as a level-specific, almost non-invasive, probe into the surving population of excited ions circulating in the ring.

Another probe is provided by laser light that can be used to pump population from a long-lived level to a short-lived one that either decays back or to another level; in 


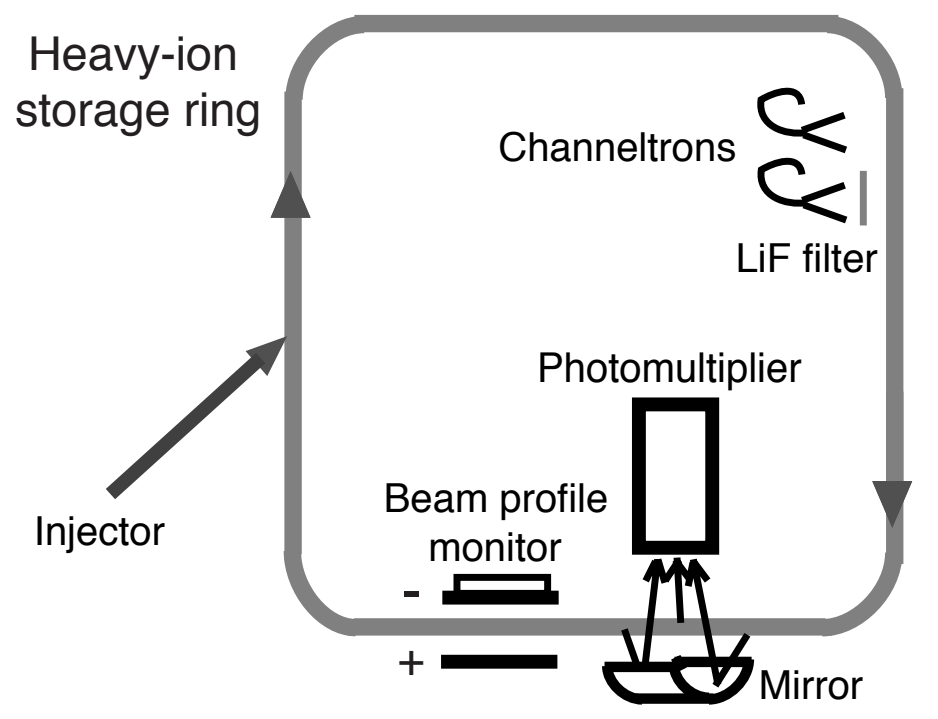

Figure 3. Lay-out of atomic lifetime experiments using passive observations at a heavy-ion storage ring.

any case the fluorescence is proportional to the population of the long-lived level. Laser excitation can be quite narrow-band, especially for co- and counterpropagating beams that combine to effect two-photon processes. The Doppler shift between these laser beams as seen by the fast moving ions can be very helpful in shifting the laser stray light out of the bandpass of the detector, or in Doppler shifting visible laser light into the near-uv for excitation. At the ESR storage ring at GSI Darmstadt, in the observation of the ground state hyperfine structure of H-like ${ }^{209} \mathrm{Bi}^{82+}$ ions, counterpropagating visible laser light of $489 \mathrm{~nm}$ was seen in the ion rest frame (at more than half the speed of light) as having a wavelength of about $243 \mathrm{~nm}[56,57,58]$. Pulsed laser excitation resulted in delayed fluorescence with a time constant near $0.3 \mathrm{~ms}$.

Another way of employing a laser has been found at CRYRING (Stockholm), were, however, mostly singly charged ions are being studied. The laser pulse is so bright that it pumps all the population out of the long-lived level. A decay curve is built up over many experimental cycles; in each cycle the laser-induced fluorescence signal at a given time after injection is obtained as a single data entry that tells how much excited level population was left. In order to study repopulation processes (much more likely at the relatively low ion beam energies used at Stocholm than at the energies used elsewhere), the population may be quenched early, and then probed again later, checking whether there is a significant amount of ions in a given metastable level again [59].

In all these decay curve experiments, the observed decay rate represents the sum of the radiative decay rate and the ion loss rate from the stored ion beam, that is the decrease of the ion beam current over time. The beam current can be monitored in various ways; a practical one is the use of the beam profile monitor that observes the spatial distribution of particles of the residual gas that have been ionized in collisions with the fast ion beam. A planar electric field draws these low-energy recoil ions to 
a microchannelplate detector with a position-sensitive read-out. The overall signal rate depends on the ion beam current and will dwindle with the beam. The slope of the current signal reveals the ion loss rate, since only one charge state species (one isotope, one beam velocity) is stored in the ring. After this technique had produced highly accurate lifetime data on a number of relatively low charge state ions, the quest continued with higher charge state ions. The low charge state ions had usually be produced by gas stripping in the injector accelerator serving the Heidelberg storage ring. Higher charge states required the use of a foil stripper. The latest experiments suggest that then the bane of beam-foil spectroscopy, cascades from enormously longlived higher-lying levels [60], may affect lifetime measurements at the storage ring, too.

\subsection{Classical ion traps: Kingdon, Penning, Paul}

The heavy-ion storage ring is, in fact, a large ion trap, with ions of energies of a few dozen $\mathrm{keV}$ to many $\mathrm{MeV}$. There also are ion traps for less energetic ions. These are, for example, the cylindrical-symmetric electrostatic trap (Kingdon), the Penning trap in which a strong magnetic field constrains the ion motion across the field, and a static voltage on some drift tubes limits the ion motion along the magnetic field lines. In the radiofrequency (Paul) trap, a ring and two cap electrodes at a static voltage and a superimposed radiofrequency field form a quadrupole field that can trap ions. There are optimum geometries of the trap electrodes (hyperboloidal surfaces), and many simpler designs (all electrodes being part of a common cylinder, or a flat arrangement) that offer benefits of access or manufacture. There also are various other designs, for example the electrostatic mirror [61, 62]. All that is needed in the present context are the common principles, not the details. Since most traps operate with conservative fields, the ions of interest have either to be produced inside the trap to stay there, or the traps have to be opened to admit particles, and then be closed in order to constrain them. A problem then is to determine how many ions are being stored, or how large the loss rate is. The Zajfman trap [61,62] relies on charge exchange processes that will ultimately lead to low charge states or even neutral particles that leak out through the electrostatic mirror towards a detector on the symmetry axis. Other traps are being opened after a while, and a detector outside captures a fraction of the ejectiles from which the surviving number of ions is being estimated - with very limited information on the charge state distribution in the sample. Laser probing of the population of metastable levels is an option, but only in ions that are not highly charged. There are limits because of the energy level structure, but also because it is difficult to cool highly charged ions sufficiently to take advantage of narrowband lasers. If the ions are confined spatially, but their energy is not very low (and thus the velocity distribution is not very narrow), ordinary lasers will not be intense enough to cause much fluorescence.

From a combination of many such factors, lifetime measurements on highly charged ions in classical ion traps are few, and in hindsight many of them appear to suffer from systematic errors larger than recognized. Most of these errors are being taken 
into account in a new generation of electrostatic ion storage rings (ELISA at Aarhus, DESIREE at Stockholm, CSR at Heidelberg), some of which are to operate at extremely low temperature in order to obtain a near-perfect vacuum and to suppress the influence of black body radiation. The first such devices are dedicated to low charge state ion work (including simple molecules or even large biomolecules), but in the Heidelberg CSR project, operation with highly charged ions is also foreseen, and it will include lifetime measurements, for example with the aim of finding out whether the absence of magnetic fields is important, or whether the extremely low pressure permits the measurement of very long atomic lifetimes (into the many minute range?). In a few years we will know more.

\subsection{Electron beam ion trap}

There is one variant of the classical Penning trap that is capable of operating with highly charged ions, and, in fact, it can do so with all charge states of all elements, as it breeds them internally. This is the electron beam ion trap (EBIT) [63, 64, 65] that combines the Penning trap principle with a strong, extremely well collimated electron beam along the magnetic field (of typically 3 to $5 \mathrm{~T}$ field strength $\mathrm{B}$ ), thus also defining an axis of symmetry. The electron beam is compressed by the field, to a diameter of about $60 \mu \mathrm{m}$. The "Penning" parts of the trap are completed by drift tubes on different potentials that keep ions in the trap volume axially confined. The electron beam serves several purposes: the electrons collisionally ionize atoms from the ambient gas or from a gas flow injected ballistically on purpose (for example, a neutral gas stream of a density corresponding to a pressure of as low as $10^{-10}$ mbar may be crossing the electron beam trajectory under UHV conditions $\left(<10^{-11} \mathrm{mbar}\right)$ ), or low-charge ions injected along the magnetic field lines from an external MeVVA ion source. Instead of a gas injector, neutral atoms may also be provided by laser-produced ablation of an external target. These freshly produced ions are then confined by the trap fields and can be hit by fast electrons over and over again. If the electron-ion collisions are sufficiently frequent (that is why the electron beam needs to be so tightly focused) and energetic, stepwise ionization to ever higher charge states can proceed. This process is moderated by the interplay of ionization, recombination and charge-changing collisions with the residual gas (this determines the need for ultrahigh vacuum (UHV)). The charge state limit is given by the electron energy and the increasingly high ionization potentials of highly charged ions. The second job of the electron beam is a compensation of the space charge of the cloud of positive ions that is being built up in the trap. Even with a strong magnetic field for radial confinement, the ions would repel each other and move away from the location of the electron beam, if the attractive potential of the beam electrons and the space charge compensation were absent.

The electron-ion collisions ionize the target ions until the beam energy can no longer overcome the ionization potential. Therefore the highest charge state in the cloud of trapped ions can be predetermined. Since the electron beam can be varied in current 
and energy, this offers ways to make lifetime measurements.

2.5.1. Line width measurements As discussed above, the spectral resolution of some beam-foil spectroscopic observations has been high enough to measure the spectral line broadening that resulted from autoionization, a consequence of the uncertainty principle by which the extremely short level lifetime corresponds to an increased level width. The same lifetime range of a few femtoseconds applies also to electric dipole transitions of fewelectron ions in the mid- $Z$ range of the periodic table, which have transition wavelengths in the x-ray range. Measuring the line width in such ions takes several steps, beyond the provision of ions of the desired charge state. A spectrometer of sufficient resolving power is only one of the prerequistes. Ions produced by frequent collisions with multi$\mathrm{keV}$ electrons have kinetic energies in the $\mathrm{keV}$ range, and this does not change by the ions being trapped. Trapping is improved by evaporative cooling, which is best achieved by mixing in a light ion species. The Doppler broadening of spectral lines emitted by $\mathrm{keV}$ ions largely camouflages the underlying natural line width (Lorentz curve). Only when the trap was made very shallow, evaporative cooling of the stored ion cloud achieved to bring down the ion motion sufficiently to permit lines from long-lived levels to shrink substantially in width below that of short-lived levels. The pilot experiment on this topic [66] worked with $\mathrm{Cs}(\mathrm{Z}=55)$ and intercompared the widths of lines from $3 \mathrm{~s}$ and $3 \mathrm{~d}$ levels of the Ne-like ion $\mathrm{Cs}^{45+}$. Similarly to this Ne-like ion species, Graf et al [67] have evaluated EBIT measurements of the He-like ion $\mathrm{Fe}^{24+}$. Here the resonance line ' $\mathrm{w}$ ' was investigated in comparison to the intercombination line ' $y$ ' and the forbidden line ' $z$ '. Both of these studies yielded lifetime results within a factor of two of well-established predictions. It is not yet clear what limits the accuracy; one of the problems is the general difficulty of the analysis of a Voigt line profile (convolution of Lorentzian and Gaussian profiles). In order to determine the Lorentzian part well, the low-intensity line wings are important that, however, overlap with those of the neighbouring lines.

2.5.D. Magnetic trapping When the electron beam contributes to the trapping, an EBIT is said to operate in electronic trapping mode. However, when the beam is switched off, a Penning trap remains, and EBIT is said to operate in magnetic trapping mode [68], with ion storage being effected for many seconds (as has been ascertained by ion cyclotron resonance frequency observations at LLNL) [68, 69]. The vast majority of lifetime measurements at electron beam ion traps has been obtaining decay curves in the magnetic trapping mode. The basic arrangement is simple. A detector system views the center of the trap through slots in the drift tubes. For detectors that are sensitive to stray magnetic fields (most EBITs operate with superconducting magnets), such as photomultiplier tubes, an optical system images the trap onto the detector which sits at some distance [70] (figure 4). The electron beam is switched on for a few hundred milliseconds or so until the proper charge state ions have been bred and the signal level has risen to show this. Then the electron beam is switched off for the duration of several times the atomic lifetime of interest, so that the delayed emission signal can reach the 


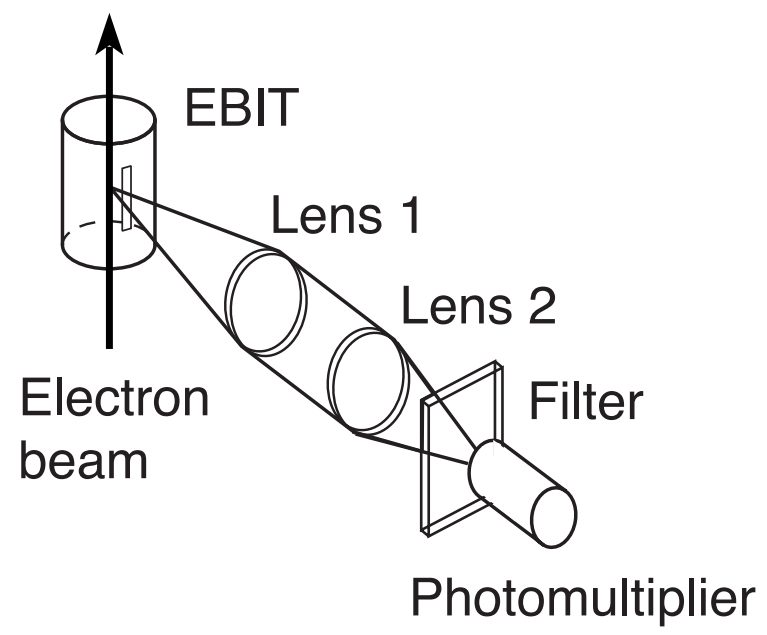

Figure 4. Optical observation at an EBIT (adapted from [70]).

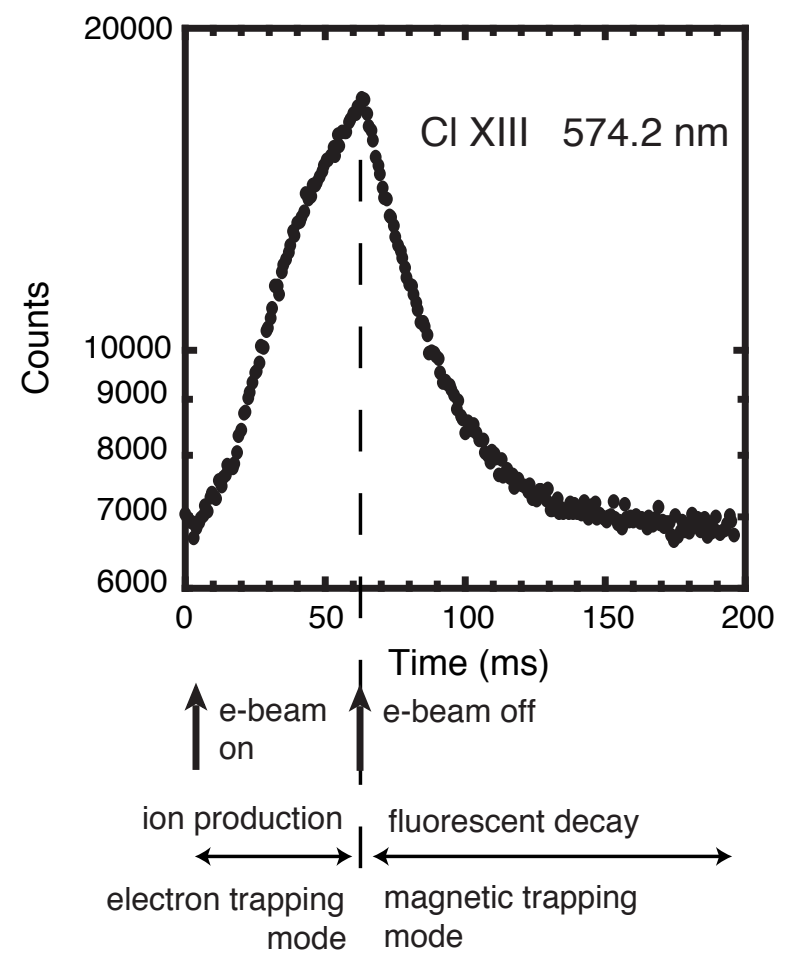

Figure 5. Timing pattern of a measurement with the 'stovepipe' spectrometer (adapted from [70]).

background level (figure 5). Then the ions are purged from the trap (by lowering the potential of one of the drift tubes, and the cycle is repeated, thousands of times.

This basic scheme has experienced many variations; for some measurements of the metastable level in He-like ions, the electron beam has not been switched off completely, but only lowered in energy below the excitation threshold [71]. Some Heidelberg 
measurements strongly reduced the electron beam current, but did not fully cut it. The purge does not have to occur in each cycle, but not much is won by saving ions from a purge: the ion cloud expands once the electron beam is gone, and it does not shrink back to its previous size when the beam is restored.

2.5.3. Ion loss measurements / $C X$ The optical signal decay rates observed on the ion cloud in an ion trap represent the sum of the true atomic decay rate and of all other loss processes, notably the loss of ions from the stored sample. Hence it is of paramount importance to determine the ion storage time constant (the inverse of the loss rate). Only when the loss rates are known, can the radiative transition rate be determined from the apparent decay rate. In contrast to heavy-ion storage rings, where only ions of a single charge state are being stored, so that a measure of the change of ion beam current is at the same time a measure of the change of ion number, most other ion traps may hold several ion species (elements and charge states) simultaneously. The number of ions of a given charge state may increase by charge exchange (CX) collisions of a higher charge state ion with the residual gas. This effect has been seen drastically in lifetime measurements on He-like ions of $\mathrm{Ne}[72,73]$. Those observations strongly suggest to make the charge state of interest the highest of the charge state distribution or, in other words, keep the electron beam energy low enough so that no higher charge state is produced.

Charge exchange also reduces the number of ions of the proper charge state, and it is not easy to determine the loss rate. A general signature of CX is delayed photon emission by the ion that gained an electron, most often capturing into a high $n$, high $l$ state. This excited ion will decay eventually, often in many steps. It is difficult to selectively observe the photons from typical steps along the decay chains, because the number density of CX-excited ions is small, and narrowband optical detection of spontaneous emission is fairly inefficient. For a variety of ions, the optical determination of the CX loss rate is simply not practical. Only if the decay chain reaches the x-ray range, energy dispersive detectors of large detection solid angle become applicable. Typical solidstate detectors have a resolution that does not distinguish individual charge states, and the x-ray energy detection threshold of about $1 \mathrm{keV}$ is also limiting the information that can be gained. Assuming that the CX processes largely reflect the density of the residual gas, one might resort to storing more highly charged, few-electron ions under the same vacuum conditions, and use their x-ray emission to determine the CX loss rate. This transfer of information gained on, say, ions with a K-shell vacancy to others with an open L-shell is based on the assumption that the CX cross sections follow simple scaling rules, which they are very likely to follow for ions with a constant number of electrons. The interpretation of L-shell processes in terms of K-shell processes, however, is burdenend with uncertainties. Modern microcalorimeters, in contrast to the traditional x-ray diodes, have a lower detection threshold (several hundred $\mathrm{eV}$ ) and a much higher resolution (say, $5 \mathrm{eV}$ ). With such a device, the CX signal of an individual charge state ion species can be followed, reducing the associated systematic error. 
As an alternative control measurement, the residual gas density in the trap can be varied. This should change the $\mathrm{CX}$ rate accordingly, which provides an in situ probe. Neglect of this test has been suggested [74] as a possible reason to explain the unsatisfactory results of some earlier experiments.

\subsection{Examples}

The lifetimes of more than 80 levels in multiply charged ions have by now been measured with trapping techniques, after hundreds were measured by beam-foil spectroscopy. In the process, the calculations of radial wavefunctions and of multiplet mixing, nondiagonal matrix elements, relativistic effects on wave functions and transition operators, multipole expansions of the radiation field, configuration interaction, hyperfine interaction, multipole mixing, approximations of the Breit operator, autoionization decay, spin-orbit, spin-spin, spin-other-orbit interactions, and whatever else were tested for their influence on atomic level lifetimes. More often than not, several entries of this list act in combination, and their individual influences cannot always be determined by experiment. The best chance to distinguish the contributions usually is the study of isoelectronic sequences, because of the different $Z$-dependences of the individual effects. The following examples are roughly ordered by increasing number of electrons, be they the total number or the number in the valence shell.

Electric dipole transitions in He-like ions have been studied up to $U(Z=92)$, including lifetime measurements that were almost exclusively performed by beam-foil techniques. In He-like U, the x-ray decay of the $1 . s 2 s{ }^{3} \mathrm{~S}_{1}$ level is very fast; however, there is a slower in-shell E1 cascade from one of the $1 s 2 p$ levels. Transition rates depend on the transition energy, and in this case the transition energy contains a major fraction from QED effects, the Lamb shift. Thus a lifetime measurement has revealed the then most accurate information on the $n=2$ Lamb shift in a He-like very highly charged ion $[26]$.

The aforementioned $1.2 . s{ }^{3} \mathrm{~S}_{1}$ level decays by $\mathrm{M} 1$ radiation, a process that can be understood only as a relativistic effect on the transition operator. In fact, before the decay was actually recognized in astrophysical spectra [75], it had - on theoretical grounds - been declared not to exist. Such an M1 transition rate scales with $Z^{10}$, and it is then no surprise that the level lifetime is too short to be measured in $\mathrm{U}^{90+}$, although the calculated level lifetime in neutral He is of the order of $6000 \mathrm{~s}$. Nevertheless, this decay has been studied over a lifetime range of 15 orders of magnitude, up to $\mathrm{Xe}^{52+}$ where the lifetime is only a few picoseconds [76] (figure6). To cover all that range, the various techniques described above were applied in turn. The measurement on He [77] exploited the emission from a well diagnosed gas discharge and thus is not really a lifetime measurement technique. The experiments on $\mathrm{Li}^{+}$employed a classical ion trap [78] and DR resonances in a heavy-ion storage ring [55]. Measurements on Be through N were done at the Heidelberg heavy-ion storage ring, recording the ion beam composition via the residual gas ionization signal [79] or monitoring the DR signal [80]. Experiments 


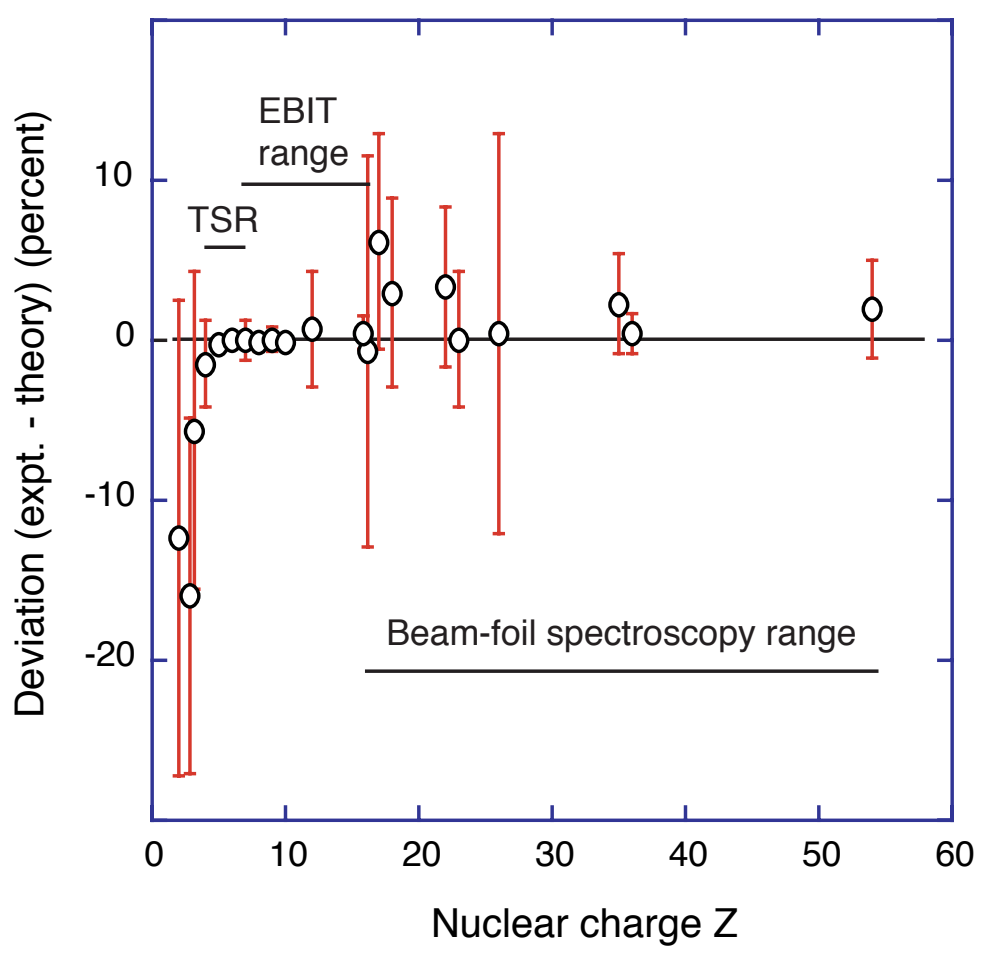

Figure 6. Iso-electronic trend of the transition rate data for the $1 \mathrm{~s}^{2}{ }^{1} \mathrm{~S}_{0}-1 \mathrm{~s} 2 \mathrm{~s}{ }^{3} \mathrm{~S}_{1}$ magnetic dipole (M1) transition in He-like ions. Data points for $Z=4$ to 7 are fom the Heidelberg heavy-ion storage ring TSR, data for $Z=7$ to $Z=10$ have been obtained at the Livermore electron beam ion trap EBIT-II. Data on heavier ions are from the use of (mostly fast) ion beams. All experimental data have been scaled by normalization to the results of the fully relativistic calculation by Johnson et al [84], and only the deviations from this prediction are displayed.

at the Livermore EBIT obtained lifetime data on He-like ions from $\mathrm{N}$ through $\mathrm{S}$ (see $[48,71,72,81]$ ), which overlaps with the range in which beam-foil experiments have been tried. At the lower end of this range $(Z=16-18)$, the lifetimes range from 200 to $700 \mathrm{~ns}$, and it is here that a slow beam experiment (on Ar) was appropriate at the time [45], because it suffered from other systematic errors than the fast beam measurements, helping to clarify the situation. This clarification process included the discovery of sign errors in theory, and eventually theory progressed so far that the best calculations differ by less than $0.1 \%$ in their prediction of this M1 transition rate [82, 83, 84]. Presently, experiment cannot afford the extreme accuracy needed to discriminate between the leading calculations for the He isoelectronic sequence. However, for now, we may consider theory to be successful in describing the two-electron ions, and then we have a ruler against which to test experimental techniques. If a measurement - in the case of this particular isoelectronic sequence - does not agree with calculation, there is likely a problem with the experiment.

The $n s-n p$ resonance line series of the alkalis evolve into a branch $\left(n p_{1 / 2}\right)$ that up to high $Z$ remains almost non-relativistic, whereas the $Z^{4}$ dependence of the fine structure 
splittings moves the $n p_{3 / 2}$ level away and thus the components of the fine structure doublet apart. At high $Z$, the $n s-n p$ wavelengths differ by more than a factor of two, and the lifetimes by a high multiple. The high- $Z$ beam-foil lifetime results with their typical 5 to $10 \%$ uncertainties are individually less meaningful than the extremely accurate lifetime data obtained on neutral Li and $\mathrm{Na}[86,87,88]$. Nevertheless, such a level of accuracy is sufficient to test many of the isoelectronic trends. For example, it was found that relativistic corrections to the matrix elements [85] were necessary to match theory and experiment in something as seemingly simple as the Li isoelectronic sequence (see [28]).

Also in the $\mathrm{Li}$ isoelectronic sequence, the $1 . s 2 p^{2}{ }^{4} \mathrm{P}_{J}(\mathrm{~J}=1 / 2,3 / 2,5 / 2)$ levels feature in-shell E1 decays to the $1 s 2 s 2 p{ }^{4} \mathrm{P}_{J}^{\circ}(\mathrm{J}=1 / 2,3 / 2,5 / 2)$ levels the rate of which scales linearly with $Z$. There also are higher multipole order x-ray decays to the $1 s^{2} 2 p$ doublet levels (the rates increase with high powers of $Z$ ), and autoionization processes that depend on spin-orbit, spin-other orbit, and spin-spin interactions (and $Z$-dependences such as $Z^{3}$ or $Z^{4}$ ). Over the years, measurements on ions from neutral Li (where almost only the in-shell radiative decay matters) to $\mathrm{Mg}, \mathrm{Al}$, and $\mathrm{Si}[89,90]$ have been made, and the isoelectronic trends of the level lifetimes helped to unravel the individual contributions [91]. It was found, for example, that the in-shell E1 transition rate was underestimated by some $20 \%$ in some early calculations; nowadays, some calculations can do much better.

Be-like ions (four electrons in total, two of them in the valence shell, see also the rather similar Mg-like ions, and so on) feature a wide range of transition types even among the lowest lying levels: typical E1, E1 with change of spin, M2, M1 between fine structure levels, hyperfine induced decays in some isotopes). The transition rate of the E1 resonance line $2 s^{2}{ }^{1} \mathrm{~S}_{0}-2 s 2 p{ }^{1} \mathrm{P}_{1}^{o}$ has been claimed to be calculable with an accuracy $\left(10^{-4}\right)$ [92] that by far exceeds experimental capabilities. Experiment, in fact, is hampered by the cascade from the $2 p^{2}{ }^{1} \mathrm{~S}_{0}$ level which differs in lifetime from the resonance level by about 30\%. (In Be, this displaced level lies above the ionization limit, and the cascade is absent.) Neglect of this growing-in cascade in beamfoil lifetime measurements has brought about a fair number of systematic overestimates of the $2 s 2 p{ }^{1} \mathrm{P}_{1}^{\circ}$ level lifetime by 30 to $50 \%$. Critical reviews and the technique of isoelectronic smoothing of the line strength data $[24,25]$ have helped to identify the more thoughtfully executed evaluations. Although the systematic shift error could be avoided, an evaluation of all sensibly evaluated lifetime data ( $\mathrm{Be}(\mathrm{Z}=4)$ through $\mathrm{Kr}$ $(Z=36))$ revealed that the authors opted for cautious error estimates in the 5 to $10 \%$ range while the scatter of the data corresponds to no more than a $3 \%$ error [28].

For the $2 s^{2}{ }^{1} \mathrm{~S}_{0}-2 s 2 p{ }^{3} \mathrm{P}_{1}^{o}$ intercombination transition in the same atomic system, the lifetime data used to reach from $\mathrm{Ne}(Z=10)$ with a $50 \%$ error estimate to Xe $(Z=54)$ with a $10 \%$ measurement error estimate. Many calculations were available, especially for the $\mathrm{C}^{2+}$ ion of astrophysical interest, but the results scattered by about $20 \%$ around the mean. The advent of heavy-ion storage ring lifetime measurements [93] and of measurement uncertainties below $1 \%$ changed the situation thoroughly - 
now theory has problems keeping up [28]. For the specific case of $\mathrm{C}^{2+}$, computationally extensive relativistic configuration interaction calculations (RCI) with about 200000 wave functions [95] appear to be the most accurate so far, with a quoted uncertainty of $0.5 \%$. The calculated result and the measurement disagree by slightly more than the combined errors - an incentive to continue the research at this high level of accuracy.

B-like ions are the simplest ions with fine structure intervals in the ground term. Between such fine structure levels, M1 and (much weaker) E2 transitions take place. Since the same term is involved in both levels, the transition rate does not depend on radial wave functions, but only on Racah algebra factors (atomic geometry, in the form of the so-called line strength $\mathrm{S}$ ) and the transition energy ( $\Delta E^{3}$ for $\mathrm{M} 1, \Delta E^{5}$ for E2 transitions). This simple picture applies to the single configuration, non-relativistic limit; otherwise the calculation can be much more difficult. Interestingly, theory has massive problems predicting fine structure intervals in many-electron systems. Most calculations are found to be insufficient in the end, and the calculated energy differences are replaced by the much more accurately known experimental data. Only very recently have $a b$ initio calculations yielded fine structure intervals close to experiment [94].

These excited levels in the ground configurations of many ions are very interesting for plasma diagnostics. The appearance of the lines indicates the presence of charge state, and thus the electron energy (temperature) of the plasma. The transitions are of relatively low energy, and consequently the spectral lines in the visible, near or extreme ultraviolet can be well resolved and identified with individual charge states. Moreover, the radiative transition rates compete with collisional excitation and deexcitation rates, at least in low density plasmas such as tokamaks or stellar coronae. Collisional-radiative modeling can be applied to identify line ratios that depend on the particle density of the plasma, because of radiative and collisional processes affecting the lower-level population. The level lifetimes of these levels are among the very few testable parameters of such modeling exercises. It has been great progress when a number of these lifetimes were measured, using electron beam ion traps or heavy-ion storage rings, with uncertainties of only one or a few percent. Such experiments have also included cross checks of the two techniques applied to the same atomic system [74]. At the same time it has been revealing to see the scatter of theoretical predictions of the same entities in multi-electron systems, ranging from a few percent in some cases to a factor of three or five in others.

For most applications in astrophysical or terrestrial plasma diagnostics, an uncertainty of radiative decay rates of one percent seems sufficient, because most other measurement parameters will be known with poorer precision. However, intellectually, it is intriguing to test how precise such measurements can be. Various experiments on the (electric dipole) resonance lines of alkali atoms have reached an uncertainty of about $0.1 \%$. The aforementioned measurement of the spin-changing E1 (intercombination) transition in $\mathrm{C}^{2+}[93]$ carried an error bar of $0.14 \%$. The same small uncertainty can test something unprecedented in the M1 transition rate between fine structure levels in many-electron ions. Beyond the simple picture mentioned above, that the transition 
rate depends only on the line strength and the transition energy (and the transition energy calculation may require a QED correction), QED also requires a correction of the transition operator. This correction relates to the electron anomalous magnetic moment (EAMM), or the (g-2) value. The correction increases the M1 transition rate by about $0.45 \%$ - and the Heidelberg EBIT results for $\mathrm{Ar}^{13+}[96,97]$ and $\mathrm{Fe}^{13+}[98]$ (while being compatible with less precise earlier measurements at the Livermore EBIT [99]) agree less well with the corrected than with the uncorrected predictions. Judged by the published uncertainties of experiment and calculation, the disagreement corresponds to several standard deviations. There is no tested idea yet of what might explain the discrepancy, whether theory missed something in the interplay of quantum mechanics and QED, or whether the experiments suffered from unrecognized systematic errors. Independent measurements are called for.

Among the M1 transitions in more complex systems is one in the $3 d^{4}$ configuration of Ti-like ions. Whereas regular fine structure intervals increase with the fourth power of the nuclear (or ion) charge, this particular interval varies very little. For more than 30 elements, the transition gives rise to a line in the near ultraviolet, which has been considered as a most promising plasma diagnostic tool [100]. Calculations have met limited success when trying to predict the transition wavelength accurately (see [101]); however, various calculations agree in showing a discontinuity of the level energy trend near $Z=55$. It is possible that lifetime measurements can help to elucidate this problem. So far only a single lifetime measurement [102] has been done on an ion in this isoelectronic sequence, and the accuracy of a sensitive test would need to be higher.

Higher-multipole order transition rates usually scale more steeply with the nuclear charge than the low orders. In various ions of $\mathrm{Kr}(\mathrm{Z}=36)$, M2 transitions have been found to contribute as much to the decay rates of certain levels as all others [103], whereas at low $Z$, the M2 contributions may be negligible. Some atomic clock schemes depend on high multipole order decays (E3) having a very low rate in atoms or singly charged ions. In highly charged ions, a magnetic octupole (M3) has been identified in the spectra of Ni-like ions of Th and $U$ [104], and its transition rate has more recently been measured in Ni-like $\mathrm{Xe}^{26+}$ [105]. In odd isotopes, the hyperfine interaction can mix sublevels of the $3 d^{9} 4 s^{3} \mathrm{D}_{3}$ and ${ }^{3} \mathrm{D}_{2}$ levels, thus mixing M3 and $\mathrm{E} 2$ decays. The effect has been measured at the Livermore EBIT, the experimental level lifetimes corroborating theory [106].

The recognition of hyperfine mixing as a contributor to certain decay channels happened in the 1930s and was put on a quantitative basis by Mrozowski [107]. The first atomic lifetime measurements showing the effect in He-like ions were reported from Berkeley [108], followed by systematic studies at Lund [109, 110]. The hyperfine quenched lifetime in $\mathrm{Ag}^{45+}$ ions served to determine the spectroscopically unresolved fine structure splitting of $1 s 2 p$ triplet levels near a level crossing along the isoelectronic sequence [111]. In the He isoelectronic sequence, the most striking hyperfine lifetime effect is that of the $1 s 2 p{ }^{3} \mathrm{P}_{0}^{\circ}$ level mixing with the $1 s 2 p{ }^{3} \mathrm{P}_{1}^{o}$ level which in turn is (relativistically) mixed with the $1 s 2 p{ }^{1} \mathrm{P}_{1}^{\circ}$ level, and the latter's very short level lifetime 
(say, in the sub-picosecond range for not so heavy ions) results in nanosecond lifetimes for the hyperfine quenched ${ }^{3} \mathrm{P}_{0}^{\mathrm{o}}$ levels - just right for beam-foil spectroscopy. The same physics applies to Be-like ions, but the reference level $2 s 2{ }^{1}{ }^{1} \mathrm{P}_{1}^{\mathrm{o}}$ may have a lifetime of hundreds of picoseconds, giving the $2 s 2 p{ }^{3} \mathrm{P}_{0}^{\circ}$ level a lifetime in the millisecond range - clearly any measurement of such lifetimes needs ion trapping. In $\mathrm{N}^{3+}$, the expected lifetime of the $2 s 2 p^{3} \mathrm{P}_{0}^{\circ}$ level is of the order of 20 minutes, and modeling of planetary nebula spectra agrees with this estimate [112]. The first storage ring measurement of such hyperfine quenching concerned Be-like ions of ${ }^{47} \mathrm{Ti}^{18+}$ and employed dielectronic recombination (DR) as a probe of the level population [113]. The lifetime result was much shorter (about 60\%) than predicted. In a photon detection experiment at the same heavy-ion storage ring, but studying the rather similar Mg-like ions of ${ }^{63,65} \mathrm{Cu}^{17+}$ a similar deviation from old calculations was noted [114]. However, newer calculations yield results some $20 \%$ shorter than the old ones (yes, theory does evolve), and the search for systematic errors in specific atomic systems and their excitation process is not finished yet (yes, experiments may still be incomplete).

Last, but not least, relativistic effects affect the wave functions. What in a low$Z$ atomic system may be rather similar fine structure components of an $L S$-coupling term can acquire quite different characters at high $Z$, due to $j$-dependent relativistic distortions of the wave functions. The individual fine structure levels may then differ drastically from each other in lifetime, and the branch fractions of their decays can be very different. Measurements of these branch fractions as well as of individual level lifetimes can give detailed insight into relativistic effects [115].

\section{Conclusion and prospects}

The published lifetime measurements of multiply charged ions reach from femtoseconds to seconds, the wavelength ranges from the visible (several $\mathrm{eV}$ photon energy) to the x-ray range. The short-lifetime range is close to the physical limit. For the measurement of long lifetimes, singly charged ion lifetimes have already exceeded one minute. In most cases, there is reasonable agreement between measured data and computed lifetimes. However, there often are several sets of data and predictions, not all

of which are compatible. Sometimes experiment erred and was made aware of that only by calculations, and in a number of cases theory obtained acceptable results only after good measurements had shown the way, that is, with little predictive power. Fortunately, incited by mutual challenge, atomic lifetime experiment and theory are evolving.

Selective excitation of specific levels in highly charged ions is virtually impossible to achieve by exposing neutral atoms to a radiation field. The situation is much more promising, if ions of a specific charge state can be prepared by different means, and photons are required only for the excitation of a given ion species. In this vein, selective laser excitation of ions stored in an electron beam ion trap has been tried at Oxford, at Livermore, and at Heidelberg, with rather limited success. However, at the FLASH facility at Hamburg, a Heidelberg-built EBIT exposed Li-like Fe ions to 
the ultraviolet light from a synchrotron light source, achieving selective excitation [116]. Such a scheme would also offer benefits for certain atomic lifetime measurements. On such resonance transitions as excited at Hamburg, the electron beam ion trap provides the ions that are to be excited. The measurement of their lifetimes, which are in the range of about a nanosecond, however, does not require any cycling of the trap voltages, only fast (sub-nanosecond) timing of the detected photons in relation to the very short excitation pulses from the light source. Considering the low photon yield of such experiments, they are best suited for short-lived levels, in measurements that exploit the high repetition frequency of synchrotron light sources. The measurement of long level lifetimes would suffer from a poor duty cycle imposed by the target atom lifetime. However, continuing development of ever brighter EUV and soft-x-ray light sources may change this perspective and open up yet another avenue leading to more insight about atomic structure and dynamics.

\section{Acknowledgments}

The author appreciates thankfully the hospitality and support experienced at the Livermore EBIT laboratory, as well as travel support by Deutsche Forschungsgemeinschaft (DFG). P. Beiersdorfer kindly provided advice on the manuscript. This work was performed in part at Lawrence Livermore National Laboratory under the auspices of the U.S. Department of Energy by Lawrence Livermore National Laboratory under Contract DE-AC52-07NA27344

\section{References}

[1] Träbert E 1997 in Accelerator-based Atomic Physics - Techniques and Applications, ed S M Shafroth and J C Austin (Washington: Am. Inst. Phys.) p567

[2] Cederquist H, Kisielinski M and Mannervik S 1983 J. Phys. B: At. Mol. Phys. 16 L479

[3] Cederquist H, Kisielinski M and Mannervik S 1984 J. Phys. B: At. Mol. Phys. 171969

[4] Träbert E 2000 Phys. Scr. $\mathbf{6 1} 257$

[5] Träbert E 2002 Phys. Scr. T 10088

[6] Träbert E 2002 Can. J. Phys. 801481

[7] Kay L 1963 Phys. Lett. 536

[8] Bashkin S and Meinel A B 1964 Astrophys. J. 139413

[9] Bashkin S 1964 Nucl. Instrum. Meth. 2888

[10] Bashkin S 1965 Science 1481047

[11] Kay L 1985 Nucl. Instrum. Methods B 9544

[12] Bashkin S 1985 Nucl. Instrum. Methods B 9546

[13] Betz H-D, Bell F, Panke H, Kalkoffen G, Welz M and Evers D 1974 Phys. Rev. Lett. 33807

[14] Northcliffe L C, Schilling R F 1970 Nucl. Data A 7233

[15] Astner G, Curtis L J, Liljeby L, Mannervik S and Martinson I 1976 Z. Phys. A 2791

[16] Barrette L and Drouin R 1974 Phys. Scr. 10213

[17] Träbert E, Winter H, Heckmann P H and v Buttlar H 1976 Nucl. Instrum. Methods 135353

[18] Baudinet-Robinet Y, Garnir H P, Dumont P D and Livingston A E 1976 Phys. Scr. 14224

[19] Ansbacher W, Pinnington E H and Kernahan J A 1988 Can. J. Phys. 66402

[20] Engström L and Bengtsson P 1991 Phys. Scr. 43480 
[21] Curtis L J 1968 Am. J. Phys. 361123

[22] Curtis L J, Berry H G and Bromander J 1971 Phys. Lett. A 34169

[23] Engström L 1982 Nucl. Instrum. Methods 202369

[24] Reistad N and Martinson I 1986 Phys. Rev. A 342632

[25] Träbert E 1988 Z. Physik D 9143

[26] Munger C and Gould H 1986 Phys. Rev. Lett. 572927

[27] Schweppe J, Belkacem A, Blumenfeld L, Claytor N, Feinberg B, Gould H, Kostroun V E, Levy L, Misawa S, Mowat J R and Prior M H 1991 Phys. Rev. Lett. 661434

[28] Träbert E, Curtis L J 2006 Phys. Scr. 74 C46

[29] Träbert E, Heckmann P H, Hutton R and Martinson I 1988 J. Opt. Soc. Am. B 52173

[30] Curtis L J 1989 Phys. Rev. A 406958

[31] Träbert E, Doerfert J, Granzow J, Büttner R, Brauckhoff J, Nicolai M, Schartner K-H, Folkmann F and Mokler P H 1995 Phys. Lett. A 20291

[32] Betz H D, Rothermel J, Röschenthaler D, Bell F, Schuch R and Nolte G 1982 Phys. Lett. A 91 12

[33] Lauer S, Liebel H, Vollweiler F, Schmoranzer H, Lagutin B M, Demekhin Ph V, Petrov I D and Sukhorukov V L 1999 J. Phys. B: At. Mol. Opt. Phys. 32, 2015

[34] Baudinet-Robinet Y, Garnir H P, Dumont P D and El Himdy A 1989 Phys. Scr. 39221

[35] Stoner jr. J O and Leavitt J A 1973 Optica Acta 20435

[36] Leavitt J A, Robson J W and Stoner jr. J O 1973 Nucl. Instrum. Methods 110423

[37] Bergkvist K E 1976 J. Opt. Soc. Am. 66837

[38] Jelley N A, Silver J D and Armour I A 1977 J. Phys. B: At. Mol. Phys. 102339

[39] Kay L and Sadler D A 1995 Phys. Scr. 51459

[40] Marrus R and Schmieder R W 1970 Phys. Rev. Lett. 251689

[41] Marrus R and Schmieder R W 1972 Phys. Rev. A 51160

[42] Gould H, Marrus R and Schmieder R W 1973 Phys. Rev. Lett. 31504

[43] Lin D L and Armstrong jr L 1977 Phys. Rev. A 16791

[44] Hubricht G, Träbert E and Hellmann H M 1986 Z. Physik D 4209

[45] Hubricht G and Träbert E 1987 Z. Physik D 7243

[46] Hubricht G and Träbert E 1989 Phys. Scr. 39581

[47] Toleikis S, Manil B, Berdermann E, Beyer H F, Bosch F, Czanta M, Dunford R W, Gumberidze A, Indelicato P, Kozhuharov C, Liesen D, Ma X, Marrus R, Mokler P H, Schneider D, Simionovici A, Stachura Z, Stöhlker T, Warczak A and Zou Y 2004 Phys. Rev. A 69022507

[48] Crespo López-Urrutia J R, Beiersdorfer P and Widmann K 2006 Phys. Rev. A 74012507

[49] Träbert E, Gwinner G, Wolf A, Knystautas E J, Garnir H-P and Tordoir X J. Phys. B: At. Mol. Opt. Phys. 35671 (2002)

[50] Träbert E, Calamai A G, Gwinner G, Knystautas E J, Pinnington E H and Wolf A 2003 J. Phys. B: At. Mol. Opt. Phys. 361129

[51] Träbert E, Saathoff G and Wolf A 2004 J. Phys. B: At. Mol. Opt. Phys. 37945

[52] Träbert E, Saathoff G and Wolf A 2004 Eur. Phys. J. D 30297

[53] Träbert E, Reinhardt S, Hoffmann J and Wolf A 2006 J. Phys. B: At. Mol. Opt. Phys. 39945

[54] Träbert E, Gwinner G, Wolf A, Tordoir X and Calamai A G 1999 Phys. Lett. A 264311

[55] Saghiri A A, Linkemann J, Schmitt M, Schwalm D, Wolf A, Bartsch T, Hoffknecht A, Müller A, Graham W G, Price A D, Badnell N R, Gorczyca T W and Tanis J 1999 Phys. Rev. A 69 R3350

[56] I. Klaft, S. Borneis, T. Engel, B. Fricke, R. Grieser, G. Huber, T. Kühl, D. Marx, R. Neumann, S. Schröder, P. Seelig, and L. Völker, Phys. Rev. Lett. 73, 2425 (1994).

[57] Seelig P, Borneis S, Dax A, Engel T, Faber S, Gerlach M, Holbrow C, Huber G, Kühl T, Marx D, Meier K, Merz P, Quint W, Schmitt F, Tomaselli M, Völker L, Winter H, Würtz M, Beckert K, Franzke B, Nolden F, Reich H, Steck M and Winkler T 1998 Phys. Rev. Lett. 814824

[58] Winter H, Borneis S, Dax A, Faber S, Kühl T, Marx D, Schmitt F, Seelig P, Seelig W, Shabaev 
V M, Tomaselli M and Würtz M 1998 GSI Scientific Report, p. 87

[59] Mannervik S 2003 Phys. Scr. T 10567

[60] Träbert E 1981 Phys. Scr. 23253

[61] Zajfman D, Heber O, Vejby-Christensen L, Ben-Itzhak I, Rappaport M, Fishman R and Dahan M 1997 Phys. Rev. A 55 R1577

[62] Dahan M, Fishman R, Heber O, Rappaport M, Altstein N, Zajfman D and van der Zande W J 1998 Rev. Sci. Instrum. 6976

[63] Levine M A, Marrs R E, Henderson J R, Knapp D A and Schneider M B 1988 Phys. Scr. T 22 157

[64] Levine M A, Marrs R E et al 1989 Nucl. Instrum. Meth. B 43431

[65] Marrs R E, Elliott S R and Knapp D A 1994 Phys. Rev. Lett. 724082 (1994).

[66] Beiersdorfer P, Osterheld A L, Decaux V and Widmann K 1996 Phys. Rev. Lett. 775353

[67] Graf A, Beiersdorfer P, Harris C L, Hwang D Q and Neill P A 2002 in CP645, Spectral Line Shapes: Vol. 12, Proc. 16th ICSLS (ed C A Back), (Washington: Am. Inst. Phys.)

[68] Beiersdorfer P, Schweikhard L, Crespo López-Urrutia J and Widmann K 1996 Rev. Sci. Instrum. 673818

[69] Beiersdorfer P, Beck B, Becker St and Schweikhard L 1996 Int. J. Mass Spectrom. Ion Proc. $\mathbf{1 5 7 / 1 5 8} 149$

[70] Träbert E and Beiersdorfer P 2003 Rev. Sci. Instrum. 742127

[71] Wargelin B J, Beiersdorfer P and Kahn S M 1993 Phys. Rev. Lett. 712196

[72] Träbert E, Beiersdorfer P, Brown G V, Smith A J, Gu M F and Savin D W 1999 Phys. Rev. A 602034

[73] Träbert E, Beiersdorfer P and Utter S B 1999 Phys. Scr. T 80450

[74] Träbert E, Beiersdorfer P, Gwinner G, Pinnington E H and Wolf A 2002 Phys. Rev. A 66052507

[75] Gabriel A H and Jordan C 1969 Mon. Not. R. Astron. Soc. 145241

[76] Marrus R, Charles P, Indelicato P, de Billy L, Tazi C, Briand J-P, Simionovice A, Dietrich D D, Bosch F and Liesen D 1989 Phys. Rev. A 393725

[77] Moos H W and Woodworth J R 1973 Phys. Rev. Lett. 30775

[78] Knight R D and Prior M H 1980 Phys. Rev. A 21179

[79] Träbert E, Gwinner G, Knystautas E J and Wolf A 2003 Can. J. Phys. 81941

[80] H. T. Schmidt, P. Forck, M. Grieser, D. Habs, J. Kenntner, G. Miersch, R. Repnow, U. Schramm, T. Schüssler, D. Schwalm, and A. Wolf, Phys. Rev. Lett. 72, 1616 (1994).

[81] Crespo López-Urrutia J R, Beiersdorfer P, Savin D W and Widmann K 1998 Phys. Rev. A 57 238

[82] Drake G W F 1971 Phys. Rev. A 3908

[83] Lin C D $1975 \mathrm{PhD}$ Thesis, Columbia University

[84] Johnson W R, Plante D R and Sapirstein J 1995 in Advances of Atomic, Molecular and Optical Physics, Vol. 35, edited by B Bederson and H Walther (San Diego: Academic Press) p255

[85] Curtis L J 1995 Phys. Rev. A 51251

[86] McAlexander W I, Abraham E R I, Ritchie N W M, Williams C J, Stoof H T C and Hulet R G 1995 Phys. Rev. A 51 R871

[87] McAlexander W I, Abraham E R I and Hulet R G 1996 Phys. Rev. A 54 R5

[88] Volz U and Schmoranzer H 1996 Phys. Scr. T 6548

[89] Livingston A E, Hardis J E, Curtis L J, Brooks R L and Berry H G 1984 Phys. Rev. A 302089

[90] Hellmann H and Träbert E 1985 Nucl. Instrum. Methods B 9611

[91] Träbert E 1985 Z. Physik A 32151

[92] P. Jönsson, C. Froese Fischer, and E. Träbert, J. Phys. B: At. Mol. Opt. Phys. 31, 3497 (1998).

[93] Doerfert J, Träbert E, Wolf A, Schwalm D and Uwira O 1997 Phys. Rev. Lett. 784355

[94] Vilkas M J, Ishikawa Y 2003 Phys. Rev. A 68012503

[95] Chen M H, Cheng K T and Johnson W R 2001 Phys. Rev. A 64042507

[96] Lapierre A, Jentschura U D, Crespo López-Urrutia J R, Braun J, Brenner G, Bruhns H, Fischer 
D, González-Martínez A J, Harman Z, Johnson W R, Keitel C H, Mironov V, Osborne C J, Sikler G, Soria Orts R, Shabaev V, Tawara H, Tupitsyn I I, Ullrich J, and Volotka A 2005 Phys. Rev. Lett. 95183001

[97] Lapierre A, Crespo López-Urrutia J R, Braun J, Brenner G, Bruhns H, Fischer D, GonzálezMartínez A J, Mironov V, Osborne C J, Sikler G, Soria Orts R, Tawara H, Ullrich J, Shabaev V M, Tupitsyn I I and Volotka A 2006 Phys. Rev. A 73052507

[98] Brenner G, Crespo López-Urrutia J R, Harman Z, Mokler P H and Ullrich J 2007 Phys. Rev. A 75032504

[99] Beiersdorfer P, Träbert E and Pinnington E H 2003 Astrophys. J. 587836

[100] Feldman U, Indelicato P and Sugar J 1991 J. Opt. Soc. Am. B 83

[101] Biémont E, Träbert E and Zeippen C 2001 J. Phys. B: At. Mol. Opt. Phys. 341941

[102] Serpa F G, Morgan C A, Meyer E S, Gillaspy J D, Träbert E, Church D A, Takács E 1997 Phys. Rev. A 554196

[103] Träbert E, Beiersdorfer P, Brown G V, Chen H, Thorn D B and Biémont E 2001 Phys. Rev. A 64042511

[104] Beiersdorfer P, Osterheld A L, Scofield J, Wargelin B and Marrs R E 1991 Phys. Rev. Lett. 67 2272

[105] Träbert E, Beiersdorfer P, Brown G V 2007 Phys. Rev. Lett. 98263001

[106] Yao K, Andersson M, Brage T, Hutton R, Jönsson P and Zou Y 2006 Phys. Rev. Lett. 97 183001; erratum 2007 Phys. Rev. Lett. 98269903

[107] Mrozowski S 1938 Z. Physik 108204

[108] Gould H, Marrus R and Mohr P J 1974 Phys. Rev. Lett. 33676

[109] Engström L, Jupén C, Denne B, Huldt S, Meng W T, Kaijser P, Litzén U and Martinson I 1980 J. Phys. B: At. Mol. Phys. 13 L143

[110] Denne B, Huldt S, Pihl J and Hallin R 1980 Phys. Scr. 2245

[111] Birkett B B, Briand J-P, Charles P, et al 1993 Phys. Rev. A 47 R2454

[112] Brage T, Judge P G and Proffitt C R 2002 Phys. Rev. Lett. 89281101

[113] Schippers S, Schmidt E W, Bernhardt D, Yu D, Müller A, Lestinsky M, Orlov D A, Grieser M, Repnow R and Wolf A 2007 Phys. Rev. Lett. 98033001

[114] Träbert E, Hoffmann J, Krantz, Reinhardt S and Wolf A (work in progress)

[115] Curtis L J, Federman S R, Torok K, Brown M, Cheng S, Irving R E and Schectman R M 2007 Phys. Scr. $75 \mathrm{C} 1$

[116] Epp S, Crespo López-Urrutia J R, Brenner G, Mäckel V, Mokler P H, Treusch R, Kuhlmann M, Yurkov M V, Feldhaus J, Schneider J R, Wellhöfer M, Martins M,. Wurth W and Ullrich J 2007 Phys. Rev. Lett. 98183001 